\title{
Initial assessment \& development of anaesthesia techniques for use of a novel, non-occlusive tracheal dilatation balloon allowing continuous
}

\section{oxygenation \& ventilation.}

Ross Hofmeyr ${ }^{1}$, Jessica McGuire ${ }^{2}$, Kenneth Park ${ }^{3}$, Matthew Proxenos $^{3}$, Peter Marwick ${ }^{4}$, Darlene Lubbe ${ }^{2}$

${ }^{1}$ Department of Anaesthesia and Perioperative Medicine, ${ }^{2}$ Department of Otolaryngology, University of Cape Town; ${ }^{3}$ Research and Development Department, DISA Life Sciences Pty Ltd, Cape Town; ${ }^{4}$ Department of Anaesthesiology \& Intensive Care, Stellenbosch University, Cape Town, South Africa

\section{Background and Goal}

Tracheal stenosis poses clinical challenges for both surgeon and anaesthesiologist. Frequently presenting as an airway emergency, it is difficult and costly to treat. Endoscopic balloon dilatation is an effective, low-risk alternative to reconstruction. However, patients may require repeat dilatation, and optimal management requires a shared airway with good communication between the anaesthesiologist and surgeon. Conflict arises due to the need for ongoing oxygenation/ventilation, and adequate duration of dilatation. A major limitation is complete occlusion of the airway by the balloon. We describe preliminary laboratory, animal and human studies assessing anaesthesia techniques with a novel, non-occlusive tracheal dilatation balloon.

\section{Materials and Methods}

Assessment was undertaken with ethical approval and informed consent in three phases. Phase 1: An airway manikin was used to familiarize the anaesthesiologist-otolaryngologist team with traditional placement through a rigid bronchoscope. Thereafter, use through an endotracheal tube (ETT) and supraglottic airway (SGA) by the anaesthesiologist under flexible endoscopic guidance were simulated. Phase 2: Balloons were deployed through ETTs during continuous ventilation in eight anaesthetised sheep while measuring airway pressures, capnography, pulse oximetry and tidal volumes. Phase 3: 20 patients with tracheal stenosis were enrolled in a prospective clinical trial. Data recorded included pre- and post-dilation grading of stenosis, airway pressures and volumes, capnography, pulse oximetry and adverse events.

\section{Results and Discussion}

Manikin testing confirmed feasibility of deployment and ventilation, and allowed development of skills by the anaesthesiologist-surgeon team. During animal testing, ventilation was maintained at all times without clinically relevant changes. All human patients were successfully ventilated during dilatation, with no hypoxia or significant adverse events. Several patients who were deemed inoperable by other methods with multilevel tracheal and bronchial stenosis were successfully treated.

\section{Conclusion}

This nonocclusive balloon allows tracheal dilatation without interruption of ventilation, conferring a significant safety advantage. It facilitates endoscopically-guided techniques, simplifying the anaesthetic management but requiring enhanced skills and cooperation by the anaesthesiologistsurgeon team. Further work will elucidate the safety and longterm efficacy.

\section{(8) OpenAirway}

Scan this QR code to access this poster online, where you can download a PDF, watch or link to the video files.
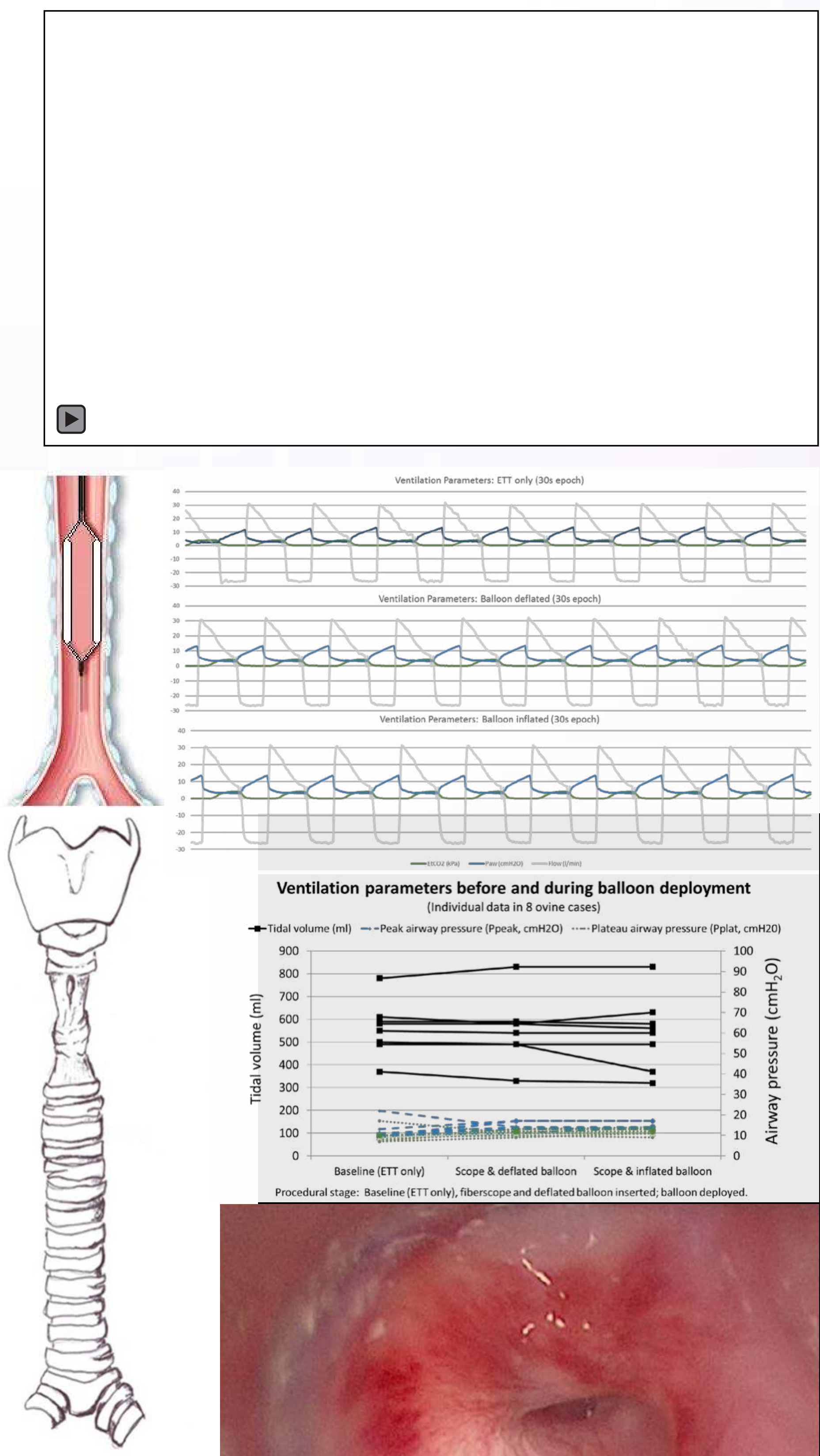

Ventilation parameters before and during balloon deployment (Individual data in 8 ovine cases)
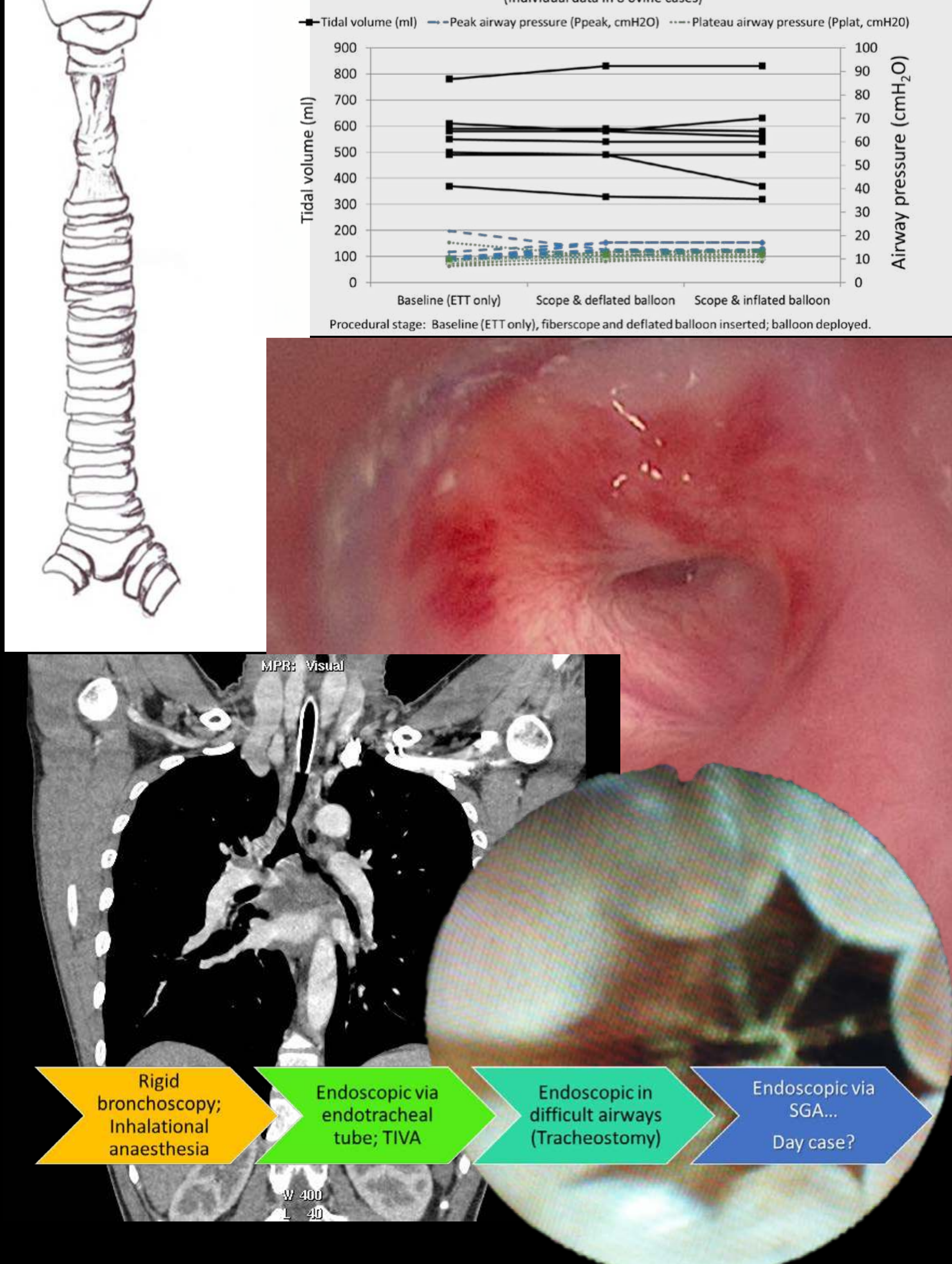

Top: Video recording of non-occlusive tracheal dilatation balloon in use in a tracheal stenosis, showing visible gas movement with ventilation through the central lumen.
DEPARTMENT OF ANAESTHESIA \& PERIOPERATIVE MEDICINE UNIVERSITY OF CAPE TOWN 